\title{
Sucesso do resfriamento e congelamento de sêmen de pirapitinga Brycon nattereri
}

[Sucess of cooling and freezing of pirapitinga (Brycon nattereri) semen]

\author{
A.V. Oliveira, A.T.M. Viveiros*, A.N. Maria, R.T.F. Freitas, Z.A. Izaú \\ Universidade Federal de Lavras \\ Caixa Postal 3037 \\ 37200-000 - Lavras, MG
}

\begin{abstract}
RESUMO
Avaliaram-se protocolos de resfriamento e de criopreservação do sêmen de pirapitinga (Brycon nattereri) utilizando-se sêmen diluído em $\mathrm{NaCl} 154 \mathrm{mM}, \mathrm{NaCl} 200 \mathrm{mM}$, Saad e BTS ${ }^{\circledR}$ e resfriado por sete dias. Cinco diluidores (glicose $277 \mathrm{mM}, \mathrm{NaCl} 154 \mathrm{mM}, \mathrm{NaCl} 200 \mathrm{mM}$, Saad e BTS ${ }^{\circledR}$ ) foram combinados com dois crioprotetores (DMSO - dimetilsulfóxido e metilglicol) e usados como meio de congelamento. O sêmen diluído em cada meio foi envasado (palhetas de $0,5 \mathrm{ml}$ ) e congelado, e a motilidade espermática avaliada após o descongelamento $\left(60^{\circ} \mathrm{C}, 8 \mathrm{seg}\right)$. O sêmen foi novamente congelado em palhetas com diferentes volumes $(0,25 \mathrm{e} 0,5 \mathrm{ml})$ e descongelados em banho-maria em duas temperaturas $\left(50^{\circ}\right.$ e $\left.60^{\circ} \mathrm{C}\right)$. As maiores motilidades $(48 \%)$ foram observadas no sêmen diluído em BTS $^{\circledR}$ e resfriado por sete dias. Motilidade espermática acima de $68 \%$ foram observadas no sêmen congelado em $\mathrm{NaCl} 154 \mathrm{mM}-$ metilglicol, BTS ${ }^{\circledR}$-metilglicol, $\mathrm{NaCl}$ 200mM-DMSO e Saad-DMSO. Não houve diferença entre os volumes de palheta nem entre as temperaturas de descongelamento quanto a motilidade espermática. Assim, o sêmen de pirapitinga mantém altas taxas de motilidade quando resfriado em BTS $^{\circledR}$ por até sete dias ou congelado em NaCl 154mM-metilglicol, BTS $^{\circledR}$-metilglicol, $\mathrm{NaCl}$ 200mM-DMSO e Saad-DMSO.
\end{abstract}

Palavras-chave: peixe, diluidores, crioprotetores, resfriamento, criopreservação

\begin{abstract}
Cooling and freezing protocols of pirapitinga (Brycon nattereri) semen were evaluated using semen diluted in $154 \mathrm{mM} \mathrm{NaCl}, 200 \mathrm{mM} \mathrm{NaCl}$, Saad or BTS ${ }^{\mathrm{TM}}$, and cooled for seven days. Sperm motility was daily evaluated. Five extenders (277mM glucose, $154 \mathrm{mM} \mathrm{NaCl}, 200 \mathrm{mM} \mathrm{NaCl}$, Saad and BTS $\mathrm{m}^{\mathrm{TM}}$ ) were combined with two cryoprotectants (DMSO - dimethyl sulphoxide and methylglycol) to produce 10 cryosolutions. Semen was diluted in each cryosolutions, aspirated into $0.5 \mathrm{ml}$ straws and frozen. Sperm motility was evaluated after thawing $\left(60^{\circ} \mathrm{C}, 8 \mathrm{sec}\right)$. Then, semen was frozen in straws with different volumes $(0.25$ and $0.5 \mathrm{ml})$, and thawed under different water-bath temperatures $\left(50^{\circ}\right.$ and $\left.60^{\circ} \mathrm{C}\right)$. Higher sperm motility (48\%) was observed when semen was cooled in BTS ${ }^{\mathrm{TM}}$ for seven days. Post-thawing sperm motility above 68\% was observed when semen was frozen in $154 \mathrm{mM}$ NaCl-methylglycol, BTS ${ }^{\mathrm{TM}}$ methylglycol, 200mM NaCl-DMSO or Saad-DMSO. There was no difference on sperm motility when semen was frozen in 0.25 or $0.5 \mathrm{ml}$ straws and thawed in $50^{\circ}$ or $60^{\circ} \mathrm{C}$ water-bath. Thus, pirapitinga semen

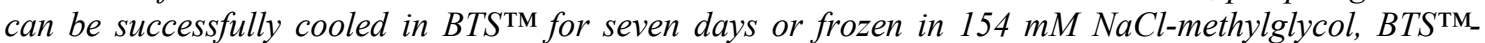
methylglycol, 200mM NaCl-DMSO and Saad-DMSO.
\end{abstract}

Keywords: fish, extenders, cryoprotectants, cooling, cryopreservation

Recebido em 12 de julho de 2006

Aceito em 27 de setembro de 2007

*Autor para correspondência (corresponding author)

E-mail: ana.viveiros@ufla.br 


\section{INTRODUÇÃO}

A pirapitinga ou parpitinga Brycon nattereri é um peixe de porte médio, que pode atingir de 40 a $50 \mathrm{~cm}$ de comprimento e apresenta dorso escuro e nadadeiras amareladas. Em geral, o macho é sensivelmente menor e possui aspereza da nadadeira anal, resultante de pequenas espículas que aparecem na época da reprodução. Essa espécie se reproduz nos meses frios e em águas rasas. A sua utilização na piscicultura vem sendo estudada na Estação de Pesquisa e Desenvolvimento Ambiental de Volta Grande da Companhia Energética de Minas Gerais (CEMIG). A espécie não é mais encontrada em várias seções do rio Grande, devido às alterações ocorridas em seu habitat (Guia..., 2000), tais como a destruição da matas ciliares, poluição dos rios e construção de barragens e hidrelétricas. Esses fatores aliados à pesca predatória colocam a pirapitinga na lista nacional das espécies ameaçadas de extinção.

A preservação de sêmen a curto prazo consiste em manter viabilidade espermática por um período de horas ou dias, em temperaturas de refrigeração, para serem utilizados posteriormente na fertilização, podendo facilitar o manejo por dispensar a presença do macho no ato da fecundação e também aumentar a eficiência da reprodução artificial nas estações de pisciculturas. Porém, alguns cuidados são determinantes para o sucesso do resfriamento, tais como redução da temperatura, fornecimento e troca de gases, prevenção do desenvolvimento bacteriano e prevenção da dissecação (Stoss e Donaldson, 1982).

Alguns pesquisadores têm resfriado sêmen de espécies do gênero Brycon, com sucesso. O sêmen de piabanha Brycon insignis foi resfriado com sucesso por até $6 \mathrm{~h}$ (Shimoda, 2004), e os de matrinxã Brycon lundii e piracanjuba Brycon orbignyanus por até $14 \mathrm{~h}$ (Marques, 2001). Em outro estudo, o sêmen de piracanjuba foi resfriado por até sete dias apresentando taxas de motilidade acima de 37\% (Maria et al., 2006).

Quanto à criopreservação de sêmen de peixes, diversas metodologias têm sido testadas em diferentes espécies de peixe, embora os graus de sucesso sejam muito variáveis. A criopreservação em nitrogênio líquido constitui a forma viável de manutenção dos gametas masculinos por longos períodos. Também é considerada uma estratégia eficaz para a conservação do patrimônio genético de peixes brasileiros, por meio de possíveis bancos genéticos. Alguns trabalhos experimentais que envolvem a criopreservação de sêmen de espécies de peixes em extinção e nativos têm sido descritos. Em briconídeos, já foram descritos protocolos efetivos para 0 congelamento do sêmen de piracanjuba (Carolsfeld et al., 2003; Murgas et al., 2004; Maria et al., 2006) e de piabanha (Shimoda, 2004). Após extensa revisão de literatura, não foi possível encontrar relatos de um protocolo para o congelamento do sêmen de pirapitinga.

Este trabalho teve o objetivo de desenvolver um protocolo de preservação do sêmen de pirapitinga $B$. nattereri a curto prazo, pelo processo de resfriamento, e a longo prazo, por meio da criopreservação.

\section{MATERIAL E MÉTODOS}

O trabalho foi realizado em julho de 2005, utilizando-se oito machos (200 a $400 \mathrm{~g}$ de peso corporal) de pirapitinga $B$. nattereri Günther, 1864, da Estação de Piscicultura da CEMIG, unidade de Itutinga. Os machos que apresentaram sêmen sob leve pressão abdominal receberam dosagem única de extrato de hipófise de carpa $(5 \mathrm{mg} / \mathrm{kg}$ peso corporal), intramuscular. Após oito horas da aplicação, o sêmen foi coletado diretamente em tubo estéril. Imediatamente após a coleta, uma amostra de sêmen de cada macho foi observada sob microscópio óptico e aquelas que apresentavam motilidade (auto-ativação) por contaminação, por água ou urina, foram descartadas. $\mathrm{O}$ sêmen de cada macho foi coletado separadamente em tubos de ensaios graduados e armazenados a $4-6^{\circ} \mathrm{C}$, para a execução dos trabalhos. Do sêmen coletado de cada macho, amostras foram retiradas e utilizadas no resfriamento e no congelamento.

Após a coleta do sêmen, foram verificadas as seguintes características seminais: volume de sêmen; concentração espermática estimada por meio de uma câmara hematimétrica Neubauer improved; motilidade espermática avaliada subjetivamente por somente um técnico, por meio de microscópio óptico e estimada em porcentagem de espermatozóides móveis em 
relação ao total observado, após ativação de uma parte de sêmen para cinco partes de $\mathrm{NaCl} 50 \mathrm{mM}$ (Maria et al., 2006).

No experimento 1 foi analisado o efeito de diferentes diluidores na preservação da motilidade do sêmen resfriado $\left(4-6^{\circ} \mathrm{C}\right)$ por até sete dias. $\mathrm{O}$ experimento foi realizado em um delineamento em blocos ao acaso com parcela subdividida. O sêmen de cada um dos machos $(\mathrm{n}=4)$ foi diluído, 1:10 (sêmen:volume total), em um dos seguintes diluidores: 1 - $\mathrm{NaCl} 154 \mathrm{mM}$ (solução fisiológica de $\mathrm{NaCl}$ 0,9\%; Maria et al., 2006); 2 - NaCl 200mM (Maria et al., 2006); 3 solução imobilizadora de Saad $(\mathrm{NaCl} 200 \mathrm{mM}+$ Tris; Linhart et al., 1993); 4 - Beltsville Thawing Solution $\left(\mathrm{BTS}^{\circledR}\right)$, Minitub (\%): glicose 80,0; citrato de sódio 12,7; EDTA 2,7; $\mathrm{NaHCO}_{3}$ 2,7; $\mathrm{KCl} 1,5$; sulfato de gentamicina 0,5 (Maria et al., 2006) e 5 - controle: sêmen in natura, sem diluição.

Cada tratamento foi mantido em um tubo de ensaio de 5ml, devidamente identificado e armazenado na geladeira a $4-6^{\circ} \mathrm{C}$. A motilidade espermática foi avaliada diariamente até sete dias após a diluição, conforme descrito para o sêmen recém-coletado.

No experimento 2 foi analisado o efeito de diferentes meios de congelamento (diluidores + crioprotetores) sobre a motilidade do sêmen descongelado. $\mathrm{O}$ experimento foi conduzido em um delineamento em blocos ao acaso. O sêmen de cada um dos machos $(n=4)$ foi diluído em uma das combinações de cinco diluidores $(\mathrm{NaCl}$ $154 \mathrm{mM}, \mathrm{NaCl} 200 \mathrm{mM}$, Saad, BTS ${ }^{\circledR}$ e glicose $277 \mathrm{mM}$ ) e de dois crioprotetores a $10 \%$, dimetilsulfóxido (DMSO) e metilglicol, totalizando 10 tratamentos (cinco diluidores $\mathrm{x}$ dois crioprotetores). $\mathrm{O}$ sêmen diluído foi envasado em palhetas de $0,5 \mathrm{ml}(\mathrm{n}=3$ palhetas por meio de congelamento por macho), vedadas com esfera plástica acondicionada em raques e congeladas em vapor de nitrogênio ${ }^{1}$ por um período de 24 horas. Em seguida, as palhetas foram transferidas para o nitrogênio líquido e armazenadas. $\mathrm{O}$ descongelamento das palhetas foi realizado em banho-maria, a $60^{\circ} \mathrm{C}$ por oito segundos (Maria et al., 2006). A motilidade espermática foi avaliada imediatamente após o descongelamento.

\footnotetext{
${ }^{1}$ Taylor-Wharton, modelo CP 300 - Theodore, EUA
}

No experimento 3 foi analisado o efeito de diferentes volumes de palhetas $(0,25$ e $0,5 \mathrm{ml})$ e temperaturas de descongelamento $\left(50\right.$ e $60^{\circ} \mathrm{C}$ por oito segundos) sobre a motilidade do sêmen descongelado. $\mathrm{O}$ experimento foi conduzido em um delineamento em blocos ao acaso. O sêmen de cada um dos machos $(\mathrm{n}=4)$ foi diluído $(1: 10)$ nos quatro meios de congelamento selecionados a partir do experimento 2: $\mathrm{NaCl} 200 \mathrm{mM}-\mathrm{DMSO}$, Saad-DMSO, NaCl 154 mM-metilglicol e BTS ${ }^{\circledR}$ metilglicol. Após a diluição, o sêmen foi envasado em palhetas de $0,25 \mathrm{ml}(\mathrm{n}=6$ palhetas) e $0,5 \mathrm{ml}$ ( $\mathrm{n}=6$ palhetas) e congelado de acordo com a metodologia descrita no experimento anterior. Três palhetas de cada volume para cada meio de congelamento, foram descongeladas em banhomaria em uma das duas temperaturas testadas (50 ou $60^{\circ} \mathrm{C}$ ) por oito segundos. $\mathrm{O}$ arranjo fatorial utilizado foi $4 \times 2 \times 2$ (quatro meios de congelamento, dois volumes de palhetas e duas temperaturas de descongelamento). A motilidade foi avaliada imediatamente após $\mathrm{O}$ descongelamento.

Para os dados observados (taxa de motilidade espermática), o resíduo de cada modelo foi testado para distribuição normal. Os valores que não apresentaram essa distribuição foram transformados em arc seno $\sqrt{\mathrm{X}}$ para sua normalização. Os dados foram submetidos à análise de variância e as médias comparadas pelo teste Scott-Knott a $5 \%$ de probabilidade, utilizando-se o pacote computacional SISVAR (Ferreira, 1999).

\section{RESULTADOS}

O sêmen apresentou-se branco, com pouca viscosidade, volume médio de $7,6 \pm 1,1 \mathrm{ml}$ e concentração espermática média de $30,0 \times 10^{9} \pm 5,6 \times 10^{9}$ espermatozóides $/ \mathrm{ml}$.

Todos os diluidores testados durante o resfriamento do sêmen (experimento 1) foram capazes de manter a motilidade espermática imediatamente após a diluição, no dia 0 (Tab. 1). A motilidade espermática de todas as amostras, diluídas ou não, declinou à medida que o tempo de armazenamento aumentou. Após o primeiro dia de resfriamento, as amostras diluídas em $\mathrm{BTS}^{\circledR}$ apresentaram motilidade espermática mais elevada $(\mathrm{P}<0,05)$ que as diluídas em $\mathrm{NaCl}$ 
$154 \mathrm{mM}, \mathrm{NaCl} 200 \mathrm{mM}$ e Saad, porém, semelhante ao sêmen in natura, não diluído. A partir do segundo dia de resfriamento até o sétimo dia, foi observado que amostras de sêmen diluídas em BTS $^{\circledR}$ apresentaram motilidades espermáticas mais altas $(\mathrm{P}<0,05)$, que as do sêmen in natura. As amostras diluídas em BTS ${ }^{\circledR}$ apresentaram motilidade espermática acima de $80 \%$ por até três dias, não havendo diferença $(\mathrm{P}>0,05)$ entre os períodos de resfriamento.

Tabela 1. Motilidade (\%; média \pm desvio-padrão) do sêmen de pirapitinga diluído em diferentes soluções e armazenado a $4-6^{\circ} \mathrm{C}$ por até sete dias

\begin{tabular}{lllllll}
\hline Diluidor & Dia 0 & Dia 1 & Dia 2 & Dia 3 & Dia 5 & Dia 7 \\
\hline $\mathrm{NaCl} 154 \mathrm{mM}$ & $98 \pm 5 \mathrm{aA}$ & $73 \pm 15 \mathrm{aB}$ & $66 \pm 13 \mathrm{bB}$ & $49 \pm 15 \mathrm{bC}$ & $2 \pm 2 \mathrm{bD}$ & $0 \pm 0 \mathrm{bD}$ \\
$\mathrm{NaCl} 200 \mathrm{mM}$ & $94 \pm 5 \mathrm{aA}$ & $73 \pm 10 \mathrm{aB}$ & $65 \pm 11 \mathrm{bC}$ & $48 \pm 17 \mathrm{bD}$ & $6 \pm 6 \mathrm{bE}$ & $1 \pm 1 \mathrm{bE}$ \\
$\mathrm{Saad}$ & $96 \pm 5 \mathrm{aA}$ & $81 \pm 8 \mathrm{bA}$ & $61 \pm 14 \mathrm{bB}$ & $39 \pm 19 \mathrm{bC}$ & $7 \pm 7 \mathrm{bD}$ & $3 \pm 2 \mathrm{bD}$ \\
$\mathrm{BTS}^{\circledR}$ & $98 \pm 5 \mathrm{aA}$ & $98 \pm 3 \mathrm{aA}$ & $91 \pm 3 \mathrm{aA}$ & $88 \pm 3 \mathrm{aA}$ & $70 \pm 4 \mathrm{aB}$ & $48 \pm 6 \mathrm{aC}$ \\
Sêmen não diluído & $100 \pm 0 \mathrm{aA}$ & $93 \pm 3 \mathrm{aA}$ & $73 \pm 3 \mathrm{bB}$ & $39 \pm 20 \mathrm{bC}$ & $7 \pm 7 \mathrm{bD}$ & $2 \pm 2 \mathrm{bD}$
\end{tabular}

Médias seguidas por letras distintas, minúsculas na coluna e maiúsculas na linha, diferem entre si pelo teste ScottKnott $(\mathrm{P}<0,05)$.

Saad: $\mathrm{NaCl} 200 \mathrm{mM}+$ Tris; BTS $^{\circledR}$ : Beltsville Thawing Solution, Minitub ${ }^{\circledR}$ com (\%): glicose 80; citrato de sódio 12,7; EDTA 2,7; sulfato de gentamicina 0,5; $\mathrm{NaHCO}_{3} 2,7$ e KCl 1,5.

No experimento 2, foi observou-se efeito interativo entre diluidor e crioprotetor sobre a motilidade espermática (Tab. 2). Dentre as amostras criopreservadas em meio com DMSO, o sêmen diluído em $\mathrm{NaCl}$ 200mM e Saad apresentaram taxas de motilidade pósdescongelamento mais elevadas que as do sêmen diluído em $\mathrm{NaCl} 154 \mathrm{mM}$, BTS $^{\circledR}$ e glicose $277 \mathrm{mM}$. Dentre as amostras criopreservadas em metilglicol, os diluidores que proporcionaram as maiores taxas de motilidade foram $\mathrm{NaCl} 154 \mathrm{mM}$ $\mathrm{e} \mathrm{BTS}^{\circledR}$.

No experimento 3, a análise de variância para motilidade espermática mostrou não haver diferença significativa $(\mathrm{P}>0,05)$ entre os dois volumes de palhetas utilizados no congelamento (Tab. 3) nem entre as duas temperaturas de descongelamento (Tab. 4).
Tabela 2. Motilidade (\%; média \pm desvio-padrão) do sêmen de pirapitinga diluído em diferentes meios de congelamento e de acordo com o crioprotetor

\begin{tabular}{lcc}
\hline Diluidor & DMSO & Metilglicol \\
\hline $\mathrm{NaCl} 154 \mathrm{mM}$ & $51 \pm 8 \mathrm{bB}$ & $68 \pm 11 \mathrm{aA}$ \\
$\mathrm{NaCl} 200 \mathrm{mM}$ & $68 \pm 2 \mathrm{aA}$ & $57 \pm 12 \mathrm{bB}$ \\
Saad & $72 \pm 7 \mathrm{aA}$ & $45 \pm 9 \mathrm{bB}$ \\
$\mathrm{BTS} \circledast$ & $39 \pm 10 \mathrm{cB}$ & $72 \pm 1 \mathrm{aA}$ \\
Glicose $277 \mathrm{mM}$ & $32 \pm 8 \mathrm{cB}$ & $49 \pm 16 \mathrm{bA}$ \\
\hline
\end{tabular}

Médias seguidas por letras distintas, minúsculas na coluna e maiúsculas na linha diferem entre si, pelo teste Scott-Knott $(\mathrm{P}<0,05)$.

Saad: $\mathrm{NaCl} 200 \mathrm{mM}+$ Tris; $\mathrm{BTS}^{\circledR}$ : Beltsville Thawing Solution, Minitub ${ }^{\circledR}$ com (\%): glicose 80 ; citrato de sódio 12,7; EDTA 2,7; sulfato de gentamicina 0,5 ; $\mathrm{NaHCO}_{3}$ 2,7 e $\mathrm{KCl}$ 1,5. DMSO: dimetilsulfóxido.

Tabela 3. Motilidade (\%; média \pm desvio-padrão) do sêmen de pirapitinga congelado em diferentes meios de congelamento, envasado em palhetas de 0,25 ou $0,5 \mathrm{ml}$, e descongelado a $60^{\circ} \mathrm{C}$ por oito segundos

\begin{tabular}{|c|c|c|c|}
\hline \multicolumn{2}{|c|}{ Meio de congelamento } & \multicolumn{2}{|c|}{ Palheta (ml) } \\
\hline Diluidor & Crioprotetor & 0,25 & 0,5 \\
\hline $\mathrm{NaCl} 154 \mathrm{mM}$ & Metilglicol & $57 \pm 11$ & $51 \pm 13$ \\
\hline $\mathrm{NaCl} 200 \mathrm{mM}$ & DMSO & $47 \pm 11$ & $56 \pm 8$ \\
\hline Saad & DMSO & $59 \pm 7$ & $59 \pm 8$ \\
\hline BTS $®$ & Metilglicol & $67 \pm 11$ & $66 \pm 11$ \\
\hline
\end{tabular}

Não houve diferença entre meios de congelamento e entre volumes de palheta $(\mathrm{P}>0,05)$ pelo teste $\mathrm{F}$.

Saad: $\mathrm{NaCl} 200 \mathrm{mM}+$ Tris; $\mathrm{BTS}^{\circledR}$ : Beltsville Thawing Solution, Minitub ${ }^{\circledR}$ com (\%): glicose 80; citrato de sódio 12,7; EDTA 2,7; sulfato de gentamicina 0,$5 ; \mathrm{NaHCO}_{3} 2,7$ e $\mathrm{KCl} 1$,5. DMSO: dimetilsulfóxido. 
Tabela 4. Motilidade (\%; média \pm desvio padrão; $n=4$ machos) do sêmen de pirapitinga, congelado em diferentes criosoluções, envasado em palhetas de $0,5 \mathrm{ml}(\mathrm{n}=6$ palhetas por meio de congelamento), e descongelado a 50 ou $60^{\circ} \mathrm{C}$ por oito segundos

\begin{tabular}{lcccc}
\multicolumn{2}{c}{ Meio de congelamento } & & \multicolumn{2}{c}{ Temperatura de descongelamento $\left({ }^{\circ} \mathrm{C}\right)$} \\
\hline Diluidor & Crioprotetor & & 50 & 60 \\
\hline $\mathrm{NaCl} 154 \mathrm{mM}$ & Metilglicol & & $51 \pm 15$ & $57 \pm 8$ \\
$\mathrm{NaCl} 200 \mathrm{mM}$ & DMSO & & $53 \pm 13$ & $50 \pm 8$ \\
$\mathrm{Saad} 1$ & DMSO & & $62 \pm 6$ & $56 \pm 8$ \\
$\mathrm{BTS}{ }^{\mathrm{N} 2}$ & Metilglicol & & $66 \pm 11$ & $67 \pm 9$ \\
\hline
\end{tabular}

Não houve diferença entre meios de congelamento e entre temperaturas de descongelamento $(\mathrm{P}>0,05)$ pelo teste $\mathrm{F}$.

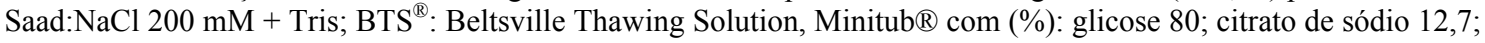
EDTA 2,7; sulfato de gentamicina 0,5; $\mathrm{NaHCO}_{3} 2,7$ e $\mathrm{KCl} 1$ 1,5. DMSO: dimetilsulfóxido.

\section{DISCUSSÃO E CONCLUSÕES}

A taxa de diluição 1:10 (sêmen:volume total) resultou em alta motilidade espermática durante a preservação do sêmen de pirapitinga. Essa mesma taxa de diluição foi utilizada na preservação do sêmen de piracanjuba e proporcionou motilidade espermática elevada em relação à taxa de diluição 1:5, tanto no resfriamento quanto no congelamento do sêmen (Maria et al., 2006).

Os diluidores testados foram selecionados a partir de experimentos realizados em preservação do sêmen de piracanjuba $B$. orbignyanus por Maria et al., (2006) e de dourado S. maxillosus por Oliveira (2006). Em pirapitinga, motilidade espermática de $48 \%$ foi observada no sêmen diluído em $\mathrm{BTS}^{\circledR}$ e preservado a $4-6^{\circ} \mathrm{C}$ por sete dias, enquanto que o sêmen in natura apresentou $39 \%$ de motilidade ainda no terceiro dia de resfriamento. Em termos de aplicação prática, amostras de sêmen mantidas resfriadas com motilidade de pelo menos $30 \%$ poderiam ser utilizadas em procedimentos de desova induzida em laboratório (Marques, 2001). O diluidor BTS $^{\circledR}$, rotineiramente utilizado no resfriamento de sêmen de suíno, também tem sido usado com sucesso na preservação de sêmen de peixes. Em piracanjuba, o sêmen, anteriormente resfriado em solução de $\mathrm{BTS}^{\circledR}$, por até seis dias, apresentou $63 \%$ de motilidade (Murgas et al., 2004). Ainda em piracanjuba, outros diluidores como $\mathrm{NaCl} 200 \mathrm{mM}$ e Saad, foram capazes de preservar o sêmen a $4-6^{\circ} \mathrm{C}$ por sete dias, com motilidade acima de 37\% (Maria et al., 2006). A solução de Saad é comumente utilizada como solução imobilizadora de motilidade espermática do bagre Europeu (Silurus glanis), superando as propriedades ativadoras proporcionadas pela contaminação com água ou urina durante a coleta de sêmen (Linhart et al., 1993). Em piau-açu (Leporinus macrocephalus), a maior motilidade espermática, $59 \%$, foi observada no sêmen diluído em $\mathrm{NaCl} 200 \mathrm{mM}$, após dois dias de resfriamento, quando comparada a outros quatro diluidores (0 a 33\%; Moraes, 2004). Em dourado, motilidade espermática acima de $30 \%$ foi observada no sêmen diluído em solução de glicose $277 \mathrm{mM}$ e preservado a $4-6^{\circ} \mathrm{C}$ por dois dias, enquanto os outros diluidores testados não conseguiram preservá-lo por mais do que um dia (Oliveira, 2006).

O sêmen in natura, não diluído, de pirapitinga, pôde ser preservado a $4-6^{\circ} \mathrm{C}$ por somente três dias e a motilidade espermática foi de $39 \%$, evidenciando a necessidade do uso de diluidores, quando resfriado por períodos iguais ou acima de três dias. Em piracanjuba, no sêmen in natura, a motilidade espermática diminui drasticamente, de $100 \%$ para $33 \%$, após 24 horas de resfriamento, sendo necessário o uso de diluidores para a sua preservação (Maria et al., 2006).

O metilglicol utilizado neste trabalho tem sido usado como crioprotetor de embriões de bovino (Takagi et al., 1993). O efeito do metilglicol foi primeiramente testado na criopreservação de sêmen, em um estudo com piracanjuba (Maria et al., 2006). Nesse estudo, o sêmen congelado em meio com metilglicol apresentou as maiores motilidades espermáticas quando comparado com o congelado em metanol ou DMSO. Em pirapitinga, entretanto, foi observada interação entre diluidores e crioprotetores. As amostras de sêmen diluídas em $\mathrm{NaCl} 154 \mathrm{mM}$ ou $\mathrm{BTS}^{\circledR}$, acrescidas de metilglicol ou em $\mathrm{NaCl} 200 \mathrm{mM}$ ou Saad, acrescidas de DMSO, apresentaram motilidade espermática semelhantes $(\mathrm{P}>0,05)$ e acima de $65 \%$ (experimento 2), após o 
descongelamento. Entretanto, quando o sêmen foi novamente congelado nesses quatro meios de congelamento, as maiores motilidades espermáticas $(66 \%)$ foram observadas no congelado em BTS $^{\circledR}$-metilglicol em relação ao congelado em outros meios (experimento 3 ). $\mathrm{O}$ BTS $^{\circledR}$, que possui $80 \%$ de glicose na sua composição, tem sido cada vez mais utilizado em criopreservação de sêmen de peixes nativos. Em piracanjuba, motilidade espermática acima de $60 \%$ foi observada no sêmen congelado em BTS $^{\circledR}$-metilglicol (Maria et al., 2006).

A glicose $277 \mathrm{mM}$ tem sido utilizada com sucesso no congelamento de sêmen de espécies nativas tais como piracanjuba (Carolsfeld et al., 2003; Murgas et al., 2004), matrinxã (Silveira, 2000), pacu (Carolsfeld et al., 2003), curimbatá, piapara e dourado (Carolsfeld et al., 2003). No entanto, neste estudo, observou-se baixa taxa de motilidade espermática quando esse diluidor foi utilizado em combinação com DMSO e com metilglicol. O sucesso no congelamento do sêmen dessas espécies tem sido obtido mediante a adição de gema de ovo no meio de congelamento, prevenindo assim os danos na membrana plasmática causada pelo congelamento. As baixas taxas de motilidade espermática obtidas nas amostras diluídas em glicose podem, provavelmente, ser atribuídas à ausência da gema de ovo.

Não foi observada diferença $(\mathrm{P}>0,05)$ na motilidade do sêmen de pirapitinga congelado em palhetas de 0,25 ou $0,5 \mathrm{ml}$ nem do sêmen descongelado em banho-maria a 50 ou $60^{\circ} \mathrm{C}$ (experimento 3). Em matrinxã, não foi observada diferença $(\mathrm{P}>0,05)$ entre a taxa de eclosão do sêmen congelado em palhetas de 0,5 ou $4,0 \mathrm{ml}$ (Silveira, 2000).

Em geral, células congeladas rapidamente, como em palhetas de $0,5 \mathrm{ml}$, devem ser mais rapidamente descongeladas quando comparadas com células congeladas em velocidade mais lenta, como em criotubos ou macropalhetas que contêm um volume maior de sêmen (Viveiros, 2005). Neste estudo, foram testadas duas temperaturas de banho-maria $\left(50^{\circ}\right.$ e $\left.60^{\circ} \mathrm{C}\right)$, que proporcionam um rápido descongelamento. Entretanto, não foi observada diferença quanto à motilidade espermática após o descongelamento. Sêmen de piracanjuba congelado em glicose-
DMSO-gema de ovo apresentou maior motilidade espermática quando descongelado a $50^{\circ} \mathrm{C}$ por 10 segundos, em relação ao sêmen descongelado a $60^{\circ} \mathrm{C}$ por cinco segundos (Murgas et al., 2003). Possivelmente, neste estudo, a diferença pode ter sido causada pelo menor tempo de imersão no banho-maria e não pela temperatura de exposição, uma vez que não foi observada diferença na motilidade do sêmen descongelado a $50^{\circ}$ ou $60^{\circ} \mathrm{C}$, por oito segundos. No descongelamento do sêmen de algumas espécies de piracemas, tem-se utilizado temperatura de banho-maria entre $26^{\circ}$ e $60^{\circ} \mathrm{C}$ e tempo de imersão entre cinco e 14 segundos (Silva, 2000; Murgas et al., 2003; Moraes, 2004; Maria et al., 2006).

No presente estudo, foi observada a velocidade de congelamento de $-37^{\circ} \mathrm{C} / \mathrm{min}$ entre as temperaturas de $+20^{\circ}$ e $-172^{\circ} \mathrm{C}$. Esse valor está dentro dos limites recomendados por Harvey e Carolsfeld (1993), que estão entre $-10^{\circ}$ a $50^{\circ} \mathrm{C} / \mathrm{min}$. No mesmo modelo de botijão, foi registrada velocidade de congelamento de $45^{\circ} \mathrm{C} / \mathrm{min}$ (Carolsfeld et al., 2003) e de $35,6^{\circ} \mathrm{C} / \mathrm{min}$ (Maria et al., 2006). Alguns fatores como o estado de conservação do botijão, o tempo para sua estabilização e a freqüência de uso podem afetar a taxa de congelamento. $\mathrm{O}$ congelamento do sêmen de pirapitinga em botijão de vapor de nitrogênio proporcionou bons resultados. Vários autores têm obtido sucesso com a utilização desse botijão no congelamento de sêmen de peixes (Ribeiro e Godinho, 2003; Murgas et al., 2003; Maria et al., 2006; Oliveira, 2006).

Baseado nesses resultados, o sêmen de pirapitinga pode ser resfriado por até sete dias a 4- $6^{\circ} \mathrm{C}$, quando diluído em $\mathrm{BTS}^{\circledR}$ ou congelado em $\mathrm{NaCl} 154 \mathrm{mM}$-metilglicol, BTS ${ }^{\circledR}$-metilglicol, $\mathrm{NaCl}$ 200mM-DMSO e Saad-DMSO, e apresentar altas taxas de motilidade espermática.

\section{AGRADECIMENTOS}

Os autores agradecem à Companhia Elétrica de Minas Gerais (CEMIG), unidade de Itutinga, pela disponibilização dos animais utilizados neste estudo, e ao estudante de Zootecnia João Fernando Albers Koch, pela ajuda na coleta de dados. 


\section{REFERÊNCIAS BIBLIOGRÁFICAS}

CAROLSFELD, J.; GODINHO, H. P.; ZANIBONI FILHO, E. et al. Cryopreservation of sperm in Brazilian migratory fish conservation. J. Fish Biol., v.63, p.472-489, 2003.

GUIA ilustrado de peixes da bacia do rio Grande. Belo Horizonte: CEMIG/CETEC, 2000. $141 \mathrm{p}$.

FERREIRA, D.F. Sistema de análise de variância (SISVAR). versão 4.3 Lavras: UFLA, 1999. (Build 43).

GUIA ilustrado de peixes da bacia do Rio Grande. Belo Horizonte: CEMIG/CETEC, 2000. $141 \mathrm{p}$.

HARVEY, B.; CAROLSFELD, J. Induced breeding in tropical fish culture. Ottawa: International Development Research Centre, 1993.

LINHART, O.; BILLARD, R.; PROTEAU, J.P. Cryopreservation of European catfish (Silurus glanis L.) spermatozoa. Aquaculture, v.115, p.347-359, 1993.

MARIA, A.N.; VIVEIROS, A.T.M.; FREITAS, R.T.F. et al. Extenders and cryoprotectants for cooling and freezing of piracanjuba (Brycon orbignyanus) semen, an endangered Brazilian teleost fish. Aquaculture, v.260, p.298-306, 2006.

MARQUES, S. Preservação a curto prazo do sêmen de teleósteos neotropicais de água doce. 2001. 83f. Dissertação (Mestrado) - Pontifícia Universidade Católica de Minas Gerais, Belo Horizonte, MG.

MORAES, G. F. Resfriamento e congelamento do sêmen de piau-açu (Leporinus macrocephalus). 2004. 68f. Dissertação (Mestrado) - Departamento de Zootecnia, Universidade Federal de Lavras, Lavras, MG.

MURGAS, L.D.S.; FRANCISCATO, R.T.; SANTOS, A.G.O. Avaliação espermática pósdescongelamento em piracanjuba (Brycon orbignyanus, Vallenciennes, 1849). Rev. Bras. Zootec., v.32, p.1810-1814, 2003.
MURGAS, L. D. S.; MILIORINI, A. B.; FRANCISCATO, R. T. et al. Viabilidade espermática do sêmen de piracanjuba (Brycon orbignyanus) resfriado a $4^{\circ} \mathrm{C}$. Rev. Bras. Zootec., v.33, p.1361-1365, 2004.

OLIVEIRA, A.V. Resfriamento e criopreservação do sêmen de dourado Salminus maxillosus $e$ de pirapitinga Brycon nattereri. 2006. 94f. Dissertação (Mestrado). Departamento de Zootecnia, Universidade Federal de Lavras, Lavras, MG.

RIBEIRO, R.I.M.A.; GODINHO, H.P. Criopreservação do sêmen testicular do teleósteo piau-açu Leporinus macrocephalus. Arq. Bras. Med. Vet. Zootec., v.55, p.75-79, 2003.

SHIMODA, E. Análise e criopreservação do sêmen da piabanha Brycon insignis Steindachner, 1877 (Pisces, Characidae). 2004. 121f. Dissertação (Mestrado). Universidade Estadual do Norte Fluminense, Campos dos Goytacazes, RJ.

SILVA, E. B. Avaliação comparativa da utilização do sêmen criopreservado e fresco na fertilização dos óvulos de curimatã Prochilodus lineatus (Valenciennes, 1836). 2000. 49f. Dissertação (Mestrado) - Universidade Federal de Santa Catarina, Florianópolis, SC.

SILVEIRA, A.N. Caracterização espermática, preservação criogênica do sêmen e fertilidade do matrinxã (Brycon cephalus). 2000. 45f. Dissertação (Mestrado) - Universidade Estadual Paulista, Botucatu, SP.

STOSS, J.; DONALDSON, E. M. Preservation of fish gametes. In: INTERNATIONAL SYMPOSIUM ON REPRODUCTION PHYSIOLOGY OF FISH, 1982, Wageningen. Proceedings... Wageningen, 1982. p.114-122.

TAKAGI, M.; BOEDIONO, A.; SAHA, S. et al. Survival rate of frozen-thawed bovine IVF embryos in relation to exposure time using various cryoprotectants. Cryobiology, v.30, p.306-312, 1993.

VIVEIROS, A. T. M. Criopreservação de sêmen de peixes. In: CONGRESSO BRASILEIRO DE REPRODUÇÃO ANIMAL, 16., 2005. Goiânia. Anais... Goiânia, 2005. (Palestras). 